\title{
Johannes Helmrath, Albert Schirrmeister, Stefan Schlelein (dir.), Medien und Sprachen humanistischer Geschichtsschreibung
}

\section{Gisela Naegle}

\section{OpenEdition}

\section{Journals}

Édition électronique

URL : http://journals.openedition.org/ifha/7561

DOI : 10.4000/ifha.7561

ISSN : 2198-8943

Éditeur

IFRA - Institut franco-allemand (sciences historiques et sociales)

Référence électronique

Gisela Naegle, « Johannes Helmrath, Albert Schirrmeister, Stefan Schlelein (dir.), Medien und Sprachen humanistischer Geschichtsschreibung ", Revue de l'IFHA [En ligne], Date de recension, mis en ligne le 13 décembre 2013, consulté le 22 septembre 2020. URL : http://journals.openedition.org/ifha/7561 ; DOI : https://doi.org/10.4000/ifha.7561

Ce document a été généré automatiquement le 22 septembre 2020.

(C)IFHA 


\title{
Johannes Helmrath, Albert Schirrmeister, Stefan Schlelein (dir.), Medien und Sprachen humanistischer Geschichtsschreibung
}

\author{
Gisela Naegle
}

Consacré aux médias et aux langues de l'historiographie humaniste, le volume présente les actes d'un colloque du Sonderforschungsbereich «transformations de l'Antiquité » de 2006. Encadré par une très courte introduction, des remarques conclusives de Caspar Hirschi et des index, il renferme neuf articles de spécialistes de philologie latine et historiens. A. Schirrmeister pose la question de la définition du genre des « descriptions de pays » (Landesbeschreibungen) humanistes et il présente des réflexions sur la constitution d'un corpus de textes. Appuyé sur les exemples de Fabius Pictor et de Polydor Vergil, Frank Wittchow examine la transformation de modèles narratifs de l'historiographie de l'Antiquité romaine dans celle de l'époque humaniste. Elisabeth Klecker traite un sujet particulièrement intéressant: Elle étudie la réception et influence méthodologique de la Historia Augusta sur les écrits de Johannes Cuspinian $(† 1529)$. La Historia est un texte antique qui « joue » avec ses lecteurs. En inventant des procédures «scientifiques» pour améliorer la crédibilité des faits rapportés, elle présente parfois des éléments purement fictifs comme vérité historique. Le but de Cuspinian est complètement différent: il recourt aux mêmes méthodes techniques, mais il recherche la fides historica et non l'éloquence. Les articles de J. Helmrath est Martin Ott intéresseront particulièrement les spécialistes de sciences auxiliaires. L'étude de J. Helmrath se réfère aux origines de la numismatique scientifique et aux fonctions de la réutilisation des portraits des empereurs des monnaies impériales dans les textes de la Renaissance. M. Ott écrit sur les inscriptions et leur appropriation par la topographie savante humaniste. Montrant l'importance et les conséquences des caractéristiques propres d'une langue, du contexte culturel et du public potentiel, la contribution de S. Schlelein est l'une des plus intéressantes du volume. L'auteur compare les techniques et méthodes de traduction de deux textes traduits - ou plutôt 
transformés - de latin en langue vernaculaire et d'un texte traduit du portugais en latin (Marineus Siculus, De rebus Hispaniae memorabilibus [latin / espagnol]; Aventin, Bayerischer Chronicon [...] kurtzer Auszug [latin- allemand] et Eanes Gomes de Azurana, Crónica da Tomada de Ceuta [portugais], version latine de Matteo de Pisano). L'étude de cas de Robert Wallisch fournit un exemple très éclairant des conséquences d'un « filtre culturel ». Dans ce cas, le récit oral d'un pèlerin chrétien indien, Josephus Indus, qui, en 1501/1502 visite Venise et Rome et rencontre le doge et le pape, est d'abord mis en écrit par un auteur anonyme vénitien. Ensuite, le texte est traduit en latin par le futur évêque d'Avellino, Arcangelo Madrigano. Josephus Indus était prêtre de l'église chrétienne dite de Thomas qui fut liée aux nestoriens de Syrie. Ainsi, il désignait le Katholikos de Syrie come «Papa» et successeur légitime de saint Pierre. Pour Madrigano, comme membre du clergé catholique, de telles formulations étaient inacceptables et il fallait trouver des solutions différentes. De même, Madrigano remplace l'auteur d'origine et le genre littéraire: sa version prétend d'être la traduction du texte d'un navigateur portugais et il la publie dans l'Itinerarium Portugallensium (1508), une sorte d'anthologie sur les découvertes des explorateurs portugais. R. Wallisch et Markus Völkel, qui écrit sur les traductions latines de textes historiographiques rédigés en langue vernaculaire, défendent l'hypothèse que de telles traductions ne peuvent pas s'expliquer par le caractère du latin comme lingua franca universelle de l'époque. M. Völkel cite un exemple convaincant : la traduction du récit du voyage au Brésil du huguenot français Jean de Léry (1534-1613). Léry traduit son propre texte de français en latin et dédie l'œuvre au Landgraf Guillaume IV de HesseKassel. Ce fait est d'autant plus étonnant parce que celui-ci avait déjà lu la version française. Ce prince passe pour francophile. Il faisait apprendre le français à son fils et l'envoyait à Paris. En outre, M. Völkel donne les exemples de textes traduits de langue vernaculaire en latin qui furent loin d'être un succès en librairie. Dans ses conclusions, C. Hirschi explique le concept méthodique des «transformations» et donne des précisions sur les buts du Sonderforschungsbereich. Il y rajoute des observations sur les relations entre les humanistes et les princes de leur temps. Dans l'ensemble, le volume est une collection très intéressante d'articles sur des questions importantes de l'époque humaniste.

\title{
INDEX
}

Thèmes : Kulturgeschichte

Index chronologique : Antike

\author{
AUTEUR \\ GISELA NAEGLE \\ Universität Gießen
}

